\title{
Reliability Equivalence Factors for Some Systems with Mixture Weibull Failure Rates
}

\author{
Abdelfattah Mustafa ${ }^{1,2}$ \\ ${ }^{1}$ Department of Mathematics, Faculty of Science, Mansoura University,Mansoura 35516, Egypt \\ ${ }^{2}$ Department of Mathematics, College of Science and Humanity Studies, Salman Bin Abdul Aziz University, \\ P. O. Box 83, Al-Kharj 11942, Saudi Arabia
}

\begin{abstract}
In this article, the failure rates of the system's components are functions of time t. We study two cases (i) the mixture of two stages of life time distribution with Weibull failure rates, (ii) the mixture of two stages failure rates with Weibull distribution. The reliability equivalence factors of some systems with identical components are obtained. Two different methods are used to improve the given systems. Numerical examples are presented to interpret how one can utilize the obtained results. Some special cases are obtained from our results.
\end{abstract}

Keywords WeibullFailure Rate Distribution, Rayleigh Distribution, Exponential Distribution, Mixture Failure Rates, Mixture Distributions, Hot Duplication, Reduction Method, Reliability Equivalence Factors

\section{Introduction}

In reliability analysis, sometimes different system designs should be compared based on a reliability characteristic such as the reliability function or mean time to failure in case of no repair. The concept of reliability equivalence factors has been introduced by[10]. The reliability equivalence factors for a single component and for two independent and identical component series and parallel systems calculated in[10,11], the author assumed that the reliability function of the system can be improved by three different methods as: (1) improving the quality of one or several components by decreasing their failure rates; (2) adding some hot redundant components to the system; and (3) adding some cold redundant component to the system. The survival function has used as the performance measure of the reliability system see[10,11]. $\operatorname{In}[12]$, the reliability equivalence factors of $n$ independent and non-identical components series system obtained, the author used the survival function and mean time to failure as characteristics to compare different system designs $[5,9,13$ -18] have applied the concept of reliability equivalence on a parallel (series) and series-parallel (parallel-series) systems with independent and identical (non- identical) components and[6] has studied the simple system of two non-identical components when the system improved by add only one component to the system by using the improving techniques.

The previous articles in reliability equivalence techniqueassumed that the system components have one type of

* Corresponding author:

abdelfatah_mustafa@yahoo.com (Abdelfattah Mustafa)

Published online at http://journal.sapub.org/ajms

Copyright (C) 2012 Scientific \& Academic Publishing. All Rights Reserved constant failure rate[8], assumed a system components have three types of constant failure rates and made a mixture of these types; see[2,3].

All articles mentioned above are about components of exponential distribution. But[19] applied the reliability equivalence factor of a parallel system with $n$ independent and identical components with Gamma life time distribution. Gamma distribution has a failure rate of function of time[7] applied the concept of reliability equivalence factor on some system with linear increasing failure rates. We hope to discuss more life time distributions, failure rates and apply the mixture approach on them to obtain the general systems.

To derive the reliability equivalence factors of a system, we use need the following definition

Definition 1. ([13])A reliability equivalence factor is a factor by which a characteristic of components of a system design has to multiplied in order to reach equality of a characteristic of this design a different design.

The reliability function and mean time to failure will be used as characteristics of the system performance. In this case the reliability equivalence will be referred as survival reliability equivalence factor, Shortly SREF, and mean reliability equivalence factor, shortly MREF, respectively.

In the current study, we shall calculate the SREF and MREF for some systems, consisting of independent and identical components. These components are assumed to be having two stages of failure rates or failure life times. The reliability of the system can be improved according to one of the following different methods:

(1) Reducing the failure rates of some of the system components. This method will be referring by the reduction method.

(2) Assuming hot duplication of some of the system 
components. This means that each component is duplicated by a hot redundant standby component. This method will be called the hot duplication method.

(3) Assuming cold duplication of some of the system components. This means that each component is duplicated by a cold redundant standby component connected with perfect (imperfect) switch. This method will be called the cold (imperfect switch) duplication method.

The methods of cold and imperfect switch duplication contain some problems in the integrations and Maple program cannot compute it. So we shall improve the study systems by using only two methods.

This paper is organized as follows. Section 2 presents the one component system with mixture of Weibull life time distributions. Section 3 gives n-components series system with mixture of two stages of Weibull failure rates. Special cases of our works are introduced.

\section{Mixture of Life Time Distributions}

We consider a system whose components fail if they enter either of two stages of failure mechanisms. The first mechanism is due to excessive voltage, and the second is due to excessive temperature. Suppose that the failure mechanism enters either the first stage with probability $\theta_{1}$ or the second stage with probability $\theta_{2}$. Let the probability density function (p.d.f.) of the first stage is $f_{1}(t)$ and the p.d.f. of the second stage is $\mathrm{f}_{2}(\mathrm{t})$. Hence the failure of a component occurs at the end of either the first or the second stage. Therefore, the p.d.f. of the failure time for a component is

$$
f(t)=\theta_{1} f_{1}(t)+\theta_{2} f_{2}(t)
$$

The reliability function of the component is

$$
R(t)=\int_{t}^{\infty} f(u) d u=\theta_{1} R_{1}(t)+\theta_{2} R_{2}(t)
$$

The hazard (failure) rate function of the component is

$$
h(t)=\frac{f(t)}{R(t)}=\frac{\theta_{1} f_{1}(t)+\theta_{2} f_{2}(t)}{\theta_{1} R_{1}(t)+\theta_{2} R_{2}(t)}
$$

Where $\theta_{1}+\theta_{2}=1$.See[2].

\subsection{The Original System}

In this section, we consider a simple system consists ofone component has two stages with Weibull failure rates as

$$
h_{i}(t)=\frac{\beta_{i}}{\alpha_{i}^{\beta_{i}}} t^{\beta_{i}-1}, \quad R_{i}(t)=\exp \left\{-\left(\frac{t}{\alpha_{i}}\right)^{\beta_{i}}\right\}
$$

where $\alpha_{i}, \beta_{\mathrm{i}}>0, t \geq 0, i=1,2$. See[1] and[2].

The life time of the stage $i$, has the Weibull distribution, from equation (2), the reliability function is given as follows

$$
R(t)=\theta_{1} \exp \left\{-\left(\frac{t}{\alpha_{1}}\right)^{\beta_{1}}\right\}+\theta_{2} \exp \left\{-\left(\frac{t}{\alpha_{2}}\right)^{\beta_{2}}\right\}
$$

Let MTTF be the system mean time to failure, which is given by

$$
\mathrm{MTTF}=\theta_{1} \alpha_{1} \Gamma\left(\frac{1}{\beta_{1}}+1\right)+\theta_{2} \alpha_{2} \Gamma\left(\frac{1}{\beta_{2}}+1\right) .
$$

where $\Gamma(x)=\int_{0}^{\infty} u^{x-1} \exp \{-u\} d u, \Gamma(\mathrm{x})=(\mathrm{x}-1)$ ! If $\mathrm{x}$ is integer, $\Gamma\left(\frac{1}{2}\right)=\sqrt{\pi}$.

\subsection{The Improved Systems}

\subsubsection{Reduction Method}

In this method, we can reducing the failure rate of the stage $i$ by the factor $\rho_{i}, 0<\rho_{i}<1, i=1,2$. Let $R_{\rho}(t)$ be the reliability function of the improved system when we reduce the failure rate of the stage $i$ by the factor $\rho_{\mathrm{i}}$. One can obtain the function $R_{\rho}(t)$ as follows

$$
R_{\rho}(t)=\theta_{1} \exp \left\{-\rho_{1}\left(\frac{t}{\alpha_{1}}\right)^{\beta_{1}}\right\}+\theta_{2} \exp \left\{-\rho_{2}\left(\frac{t}{\alpha_{2}}\right)^{\beta_{2}}\right\}
$$

From equation (7), the mean time to failure of the improved system say MTTF $_{\rho}$ becomes

$$
\operatorname{MTTF}_{\rho}=\theta_{1} \alpha_{1} \rho_{1} \Gamma\left(\frac{1}{\beta_{1}}+1\right)+\theta_{2} \alpha_{2} \rho_{2} \Gamma\left(\frac{1}{\beta_{2}}+1\right)
$$

\subsubsection{Hot Duplication Method}

Let $R^{H}(t)$ be the reliability function of the improved system assuming hot duplication. The function $R^{H}(t)$ can be obtained as

$$
R^{H}(t)=[2-R(t)] R(t)
$$

From equation (5), one can easy find $R^{H}(t)$, see[1].

Let $\mathrm{MTT} F^{H}$ denotes the mean time to failure of the system improved in this case. Using equation (9), that can be obtained by

$$
\operatorname{MTTF}^{H}=\int_{0}^{\infty} R^{H}(t) d t
$$

\section{3. $\gamma$-Fractiles}

This section presents the $\gamma$-fractiles of the original and improved systems. Let $\mathrm{L}(\gamma)$ be the $\gamma$-fractiles of the original system and $L^{H}(\gamma)$, the $\gamma$-fractiles of the improved system assuming hot duplication method.

The $\gamma$-fractiles $L(\gamma)$ and $L^{H}(\gamma)$ are defined as the solution of the two following equations, respectively,

$$
R\left(\left(\alpha_{1}+\alpha_{2}\right) L(\gamma)\right)=\gamma, \quad R^{H}\left(\left(\alpha_{1}+\alpha_{2}\right) L(\gamma)\right)=\gamma
$$

It follows from equation (5) and the first equation of (11) that $\mathrm{L}=\mathrm{L}(\gamma)$ satisfies the following equation

$$
\theta_{1} \exp \left\{-\left(\frac{\left(\alpha_{1}+\alpha_{2}\right) L}{\alpha_{1}}\right)^{\beta_{1}}\right\}+\theta_{2} \exp \left\{-\left(\frac{\left(\alpha_{1}+\alpha_{2}\right) L}{\alpha_{2}}\right)^{\beta_{2}}\right\}=\gamma
$$

From the second equation of (11), and equation (9), one can verify that $L=L^{H}(\gamma)$ satisfies the following equation

$$
2\left[\theta_{1} \exp \left\{-\left(\frac{\left(\alpha_{1}+\alpha_{2}\right) L}{\alpha_{1}}\right)^{\beta_{1}}\right\}+\theta_{2} \exp \left\{-\left(\frac{\left(\alpha_{1}+\alpha_{2}\right) L}{\alpha_{2}}\right)^{\beta_{2}}\right\}\right]+
$$


$\left[\theta_{1} \exp \left\{-\left(\frac{\left(\alpha_{1}+\alpha_{2}\right) L}{\alpha_{1}}\right)^{\beta_{1}}\right\}+\theta_{2} \exp \left\{-\left(\frac{\left(\alpha_{1}+\alpha_{2}\right) L}{\alpha_{2}}\right)^{\beta_{2}}\right\}\right]^{2}=\gamma$

Equations (12) and (13) have no closed form solution and can be solved using numerical method technique.

\subsection{Reliability Equivalence Factors}

Now we are ready to derive the reliability equivalence factors of the system. We will deduce the survival reliability equivalence factor, say SREF and mean reliability equivalence factor, say MREF of the underlying system as follows.

The first type of reliability equivalence factor, SREF say $\rho^{H}(\gamma)$, can be obtained by equating the reliability function of the improved system that obtained by improving the system according to reduction method with the reliability function of the system improved by improving the system according to hot duplication method at the level $\gamma$. Hence from equations (7) and (9), $\rho^{H}(\gamma)$, can be obtained by solving the following system of equations

$$
R_{\rho}(t)=R^{H}(t)=\gamma
$$

Let us now explain how one can deduce the second type of reliability equivalence factor. This type is MREF say $\xi^{H}$ that can be obtained by equating the mean time of the improved system that obtained by improving the system according to reduction method with the mean time to failure of the system improved by improving the system according to hot duplication method. It means that, $\xi^{H}$ can be derived from equations (8) and (10) as follows.

$$
\mathrm{MTTF}_{\rho}=\mathrm{MTTF}^{H}
$$

Equations (14), (15) can be solved numerically by using numerical method technique.

\subsection{Numerical Results}

To explain how one can apply theoretical results obtained in the previous subsections, we introduce a numerical example. In this example, we assume $\alpha_{1}=5, \beta_{1}=2, \alpha_{2}=4, \beta_{2}=3$, $\theta_{1}=0.45$, and $\theta_{2}=0.55$.

The mean time to failure of the original system is $\mathrm{MTTF}=3.95857$ and $\mathrm{MTTF}^{\mathrm{H}}=4.98433$, then $\mathrm{MTTF}<\mathrm{MTTF}^{\mathrm{H}}$.

The $\gamma$-fractiles, $\mathrm{L}(\gamma), \mathrm{L}^{\mathrm{H}}(\gamma)$ and the values of $\rho^{\mathrm{H}}(\gamma)$ are calculated using Mathematica Program system according to the previous theoretical formulae. In these calculations the level $\gamma$ is chosen to be $0.1,0.2, \ldots, 0.9$.

The $\gamma$-fractile and the survival reliability equivalence factor are given in table 1 at some values of $\gamma$.

Based on the results presented in table 1:

1. One can recognize that $\mathrm{L}(\gamma)<\mathrm{L}^{\mathrm{H}}(\gamma)$ for all studied cases, which confirms the results obtained for MTTF.

2. The hot duplication of the component increase $\mathrm{L}(0.1)$ from $0.7051\left(\alpha_{1}+\alpha_{2}\right)$ to $0.8222\left(\alpha_{1}+\alpha_{2}\right)$, see table 1 . (i) The same effect on $\mathrm{L}(0.1)$ can occur by reducing the failure rates of: (i) the first stage of the component, by the factor $\rho_{1}=0.6911$, (ii) the second stage of the component, by the factor $\rho_{2}=0.3798$, (iii) the two stages by the same factor $\rho=0.7147$.
3. In the same manner one can read the rest results.

4. The notation NA in table 1 means that the value of SREF is not available and therefore there is possible equivalence between the system improved by reduction method and that system improved by using the redundancy method at this level.

The mean reliability equivalence factors are $\xi_{1}^{H}=0.4360$, $\xi_{2}^{H}=0.2836, \xi^{H}=0.5771$, then one can conclude that, the improved system that can be obtained by improving the system according to hot duplication method, has the same mean time to failure of that system which can be obtained by doing one of the following:

(i) Reducing the failure rate of the first stage by the factor $\xi=0.4360$, (ii) reducing the failure rate of the second stage by the factor $\xi=0.2836$, (iii) reducing the failure rate of the first and second stage by the factor $\xi=0.5771$.

Table 1. $\gamma$-fractiles and $\rho^{\mathrm{H}}(\gamma)$

\begin{tabular}{c|cc|ccc}
\hline \hline$\gamma$ & $\mathrm{L}$ & $\mathrm{L}^{\mathrm{H}}$ & $\rho_{1}^{H}$ & $\rho_{1}^{H}$ & $\rho^{H}$ \\
\hline \hline 0.1 & 0.7051 & 0.8222 & 0.6911 & 0.3798 & 0.7147 \\
0.2 & 0.5889 & 0.6959 & 0.5558 & 0.4283 & 0.6684 \\
0.3 & 0.5189 & 0.6226 & 0.4221 & 0.4231 & 0.6324 \\
0.4 & 0.4641 & 0.5688 & 0.2891 & 0.3913 & 0.5937 \\
0.5 & 0.4156 & 0.5233 & 0.1543 & 0.3422 & 0.5507 \\
0.6 & 0.3689 & 0.4809 & 0.0145 & 0.2767 & 0.5020 \\
0.7 & 0.3208 & 0.4383 & NA & 0.1897 & 0.4451 \\
0.8 & 0.2669 & 0.3909 & NA & 0.0663 & 0.3747 \\
0.9 & 0.1978 & 0.3289 & NA & NA & 0.2769 \\
\hline \hline
\end{tabular}

\subsection{Special Cases}

We can calculate the reliability equivalence factors for the special cases from the present system as follows

1. if $\beta_{\mathrm{i}}=0$, equation (4), can be reduced to

$$
h_{i}(t)=\frac{1}{\alpha_{i}}, \quad R_{i}(t)=\exp \left\{-\frac{t}{\alpha_{i}}\right\}
$$

In this case, the stages have the Exponential distribution with parameter $\frac{1}{\alpha_{i}}$, see[3].

2. if $\beta_{\mathrm{i}}=2$, equation ( 4$)$, can be reduced to

$$
h_{i}(t)=\frac{2}{\alpha_{i}^{2}} t, \quad R_{i}(t)=\exp \left\{-\left(\frac{t}{\alpha_{i}}\right)^{2}\right\}
$$

In this case, the stages have the Rayleigh distribution with parameter $\frac{2}{\alpha_{i}^{2}}$,see[4].

Therefore, the reliability equivalence factor for these systems can be obtained as special cases from the studied system in this section.

\section{Mixture of Failure Rates}

In this section, we consider a system whose components fail if they enter either of two stages of failure mechanisms. The first mechanism is due to excessive voltage, and the second is due to excessive temperature. Suppose that the 
failure mechanism enters the first stage with probability $\theta_{1}$, and the failure rate is of the failure time is $h_{1}(t)$. It enters the second stage with probability $\theta_{2}$ and the failure rate of its failure time $\mathrm{h}_{2}(\mathrm{t})$. The failure of a component occurs at the end of either first stage or the second stage. Hence the failure rate of the component $i$ is

$$
h_{i}(t)=\theta_{1} h_{1}(t)+\theta_{2} h_{2}(t)
$$

The reliability function of the component $i$ is given as follows

$$
R_{i}(t)=\exp \left\{-\int_{0}^{t} h_{i}(u) d u\right\}=\exp \left\{-\left(\theta_{1} H_{1}(t)+\theta_{2} H_{2}(t)\right)\right\}
$$

where $H_{i}(t)=\int_{0}^{t} h_{i}(u) d u$ be the cumulative failure rate of the stage $i, i=1,2$. See[2].

In this section, we consider a simple system with n-identical components in series system. Each component has two stages of the hazard (failure) rate functions.

\subsection{The Original System}

In this section, we consider the stages for component $i$, have theWeibull failure rates as follows

$$
h_{i}(t)=\frac{\beta_{i}}{\alpha_{i}^{\beta_{i}}} t^{\beta_{i}-1}, i=1,2
$$

and the reliability function for the component $\mathrm{i}$ becomes

$$
R_{i}(t)=\exp \left\{-\left[\theta_{1}\left(\frac{t}{\alpha_{1}}\right)^{\beta_{1}}+\theta_{2}\left(\frac{t}{\alpha_{2}}\right)^{\beta_{2}}\right]\right\}
$$

Therefore, the reliability function of n-independent and identical components in series system is given as

$$
R(t)=\exp \left\{-n\left[\theta_{1}\left(\frac{t}{\alpha_{1}}\right)^{\beta_{1}}+\theta_{2}\left(\frac{t}{\alpha_{2}}\right)^{\beta_{2}}\right]\right\}
$$

Let MTTF be the system mean time to failure, which is given by

$$
M T T F=\int_{0}^{\infty} R(t) d t
$$

\subsection{The Improved Systems}

\subsubsection{Reduction Method}

In this method, we can reduce the mixture failure rate by reducing the stages of the failure rates by the factor $\rho_{\mathrm{i}}$, $0<\rho_{\mathrm{i}}<1, \mathrm{i}=1,2$. Let $R_{\rho, r}(t)$ be the reliability function of the system improved when reducing the failure rates of $\mathrm{r}$ components. One can obtain the function as follows

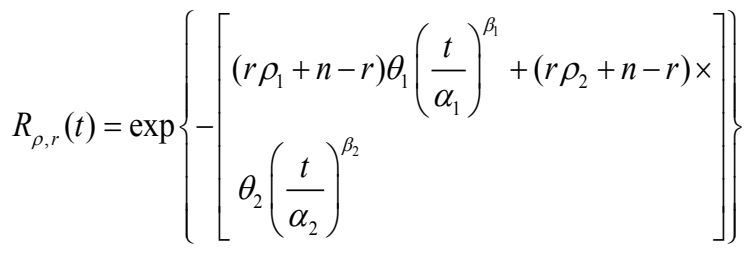

From equation (24), the mean time to failure of the improved system, say MTTF $_{\rho, \mathrm{r},}$ becomes

$$
\operatorname{MTTF}_{\rho, r}=\int_{0}^{\infty} R_{\rho, r}(t) d t
$$

\subsubsection{Hot Duplication Method}

Let $R_{m}^{H}(t)$ be the reliability function of the improved system assuming hot duplication of $\mathrm{m}$ system components. The function $R_{m}^{H}(t)$ can be obtained as

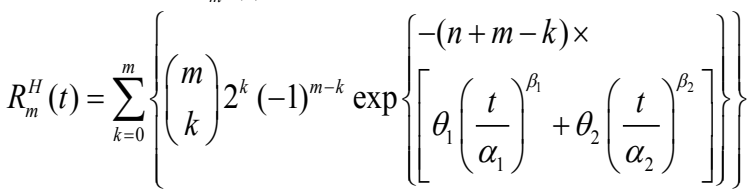

Let $\mathrm{MTTF}_{m}^{H}$ denote the mean time to failure of the system improved in this case. Using equation (22), one can obtain $\mathrm{MTTF}_{m}^{H}$ as

$$
\mathrm{MTTF}_{m}^{H}=\int_{0}^{\infty} R_{m}^{H}(t) d t
$$

The mean time to failure in $(23,25,27)$ can be calculated numerically.

\subsection{The $\gamma$-Fractiles}

In this section, we present the $\gamma$-fractiles of the original and improved systems. Let $\mathrm{L}(\gamma), L_{m}^{H}(\gamma)$ be the $\gamma$-fractiles of the original and improved system assuming hot duplication method. The $\gamma$-fractilesL $(\gamma)$ and $L_{m}^{H}(\gamma)$, are defined as the solution of the following equations, respectively,

$$
R\left(n\left(\alpha_{1}+\alpha_{2}\right) L(\gamma)\right)=\gamma, R_{m}^{H}\left(n\left(\alpha_{1}+\alpha_{2}\right) L_{m}^{H}(\gamma)\right)=\gamma
$$

It follows from equation (22) and the first equation of (28) that $\mathrm{L}$ satisfies the following equation

$$
\theta_{1}\left(\frac{n\left(\alpha_{1}+\alpha_{2}\right) L}{\alpha_{1}}\right)^{\beta_{1}}+\theta_{2}\left(\frac{n\left(\alpha_{1}+\alpha_{2}\right) L}{\alpha_{2}}\right)^{\beta_{2}}+\frac{\ln (\gamma)}{n}=0
$$

From the second equation of (28), and equation (26), one can verify that $L=L_{m}^{H}(\gamma)$ satisfies the following equation

$$
\begin{gathered}
\sum_{k=0}^{m}\left\{( \begin{array} { l } 
{ m } \\
{ k }
\end{array} ) 2 ^ { k } ( - 1 ) ^ { m - k } \operatorname { e x p } \left\{-(n+m-k)\left[\theta_{1}\left(\frac{n\left(\alpha_{1}+\alpha_{2}\right) L}{\alpha_{1}}\right)^{\beta_{1}}+\right.\right.\right. \\
\left.\left.\left.\theta_{2}\left(\frac{n\left(\alpha_{1}+\alpha_{2}\right) L}{\alpha_{2}}\right)^{\beta_{2}}\right]\right\}\right\}
\end{gathered}
$$

Equations $(29,30)$ have no closed form solution and can be solved using numerical method technique.

\subsection{Reliability Equivalence Factors}

Now we are ready to derive the reliability equivalence factors of the system. We will deduce survival reliability equivalence factor, say SREF and mean reliability equivalence factor, say MREF of the underlying system as follows.

The first type of reliability equivalence factor, SREF, say $\rho_{m, r}^{H}(\gamma)$, can be obtained by solving the following system of equations

$$
R_{m}^{H}(t)=R_{\rho, r}(t)=\gamma
$$

Let us now explain how one can deduce the second type of 
reliability equivalence factor. This type is MREF say $\xi_{m, r}^{H}$ that can be obtained by solving the following system of equations

$$
\mathrm{MTTF}_{m}^{H}=\mathrm{MTTF}_{\rho, r}
$$

Equations (31) and (32) have no closed form, which can be solved numerically by using numerical method technique.

\subsection{Numerical Results}

To explain how one can apply theoretical results obtained in the previous subsections, we introduce a numerical example. In this example, we assume three components series system with identical components, $\alpha_{1}=4, \beta_{1}=3, \alpha_{2}=3, \beta_{2}=4$, $\theta_{1}=0.45$ and $\theta_{2}=0.55$, that the failure for the stages are

$$
h_{1}(t)=0.04688 t^{2}, h_{2}(t)=0.04938 t^{3}
$$

The mean time to failure of the original system is $\mathrm{MTTF}=2.1795$ and $\mathrm{MTTF}_{m}^{H}$ are given in table 2 as follows

From table 2, one can conclude that $\mathrm{MTTF}<\mathrm{MTTF}_{m}^{H}$ for all $\mathrm{m}=1,2,3$, and increases with $\mathrm{m}$.

Table 2. The $\mathrm{MTTF}_{m}^{H}$

\begin{tabular}{c|ccc}
\hline \hline $\mathrm{m}$ & 1 & 2 & 3 \\
\hline $\mathrm{MTTF}_{m}^{H}$ & 2.3436 & 2.5525 & 2.8245 \\
\hline \hline
\end{tabular}

The $\gamma$-fractiles, $\mathrm{L}(\gamma), \mathrm{L}_{m}^{H}(\alpha)$ and the values of $\rho_{m}^{H}(\gamma)$ are calculated using Mathematica Program system according to the previous theoretical formulae.

In these calculations the level $\gamma$ is chosen to be $0.1,0.2, \ldots$, 0.9 .

The $\gamma$-fractile of the original and improved system are given in table 3 at some values of $\gamma$.

Based on the results presented in table 3 , one can recog- nize that $L(\gamma)<L_{m}^{H}(\gamma)$, for all studied cases, which confirms the results obtained for MTTF.

Table 3. The $\gamma$-fractiles

\begin{tabular}{c|c|ccc}
\hline \hline \multirow{2}{*}{$\gamma$} & \multirow{2}{*}{$\mathrm{L}$} & \multicolumn{3}{|c}{$\mathrm{L}_{m}^{H}(\alpha)$} \\
\cline { 3 - 5 } & & $\mathrm{m}=1$ & $\mathrm{~m}=2$ & $\mathrm{~m}=3$ \\
\hline 0.1 & 0.1443 & 0.1516 & 0.1591 & 0.1665 \\
0.2 & 0.1311 & 0.1391 & 0.1476 & 0.1562 \\
0.3 & 0.1213 & 0.1296 & 0.1389 & 0.1485 \\
0.4 & 0.1126 & 0.1212 & 0.1311 & 0.1418 \\
0.5 & 0.1044 & 0.1131 & 0.1236 & 0.1354 \\
0.6 & 0.0961 & 0.1047 & 0.1158 & 0.1289 \\
0.7 & 0.0871 & 0.0955 & 0.1071 & 0.1218 \\
0.8 & 0.0765 & 0.0845 & 0.0964 & 0.1132 \\
0.9 & 0.0620 & 0.0690 & 0.0807 & 0.1011 \\
\hline \hline
\end{tabular}

The values of the SREF are given in (i) table 4, when we reduce the second stage by the factor $\rho_{2}$, (ii) table 5 , when the first and second stages by the same factor $\rho$, at some values of $\gamma$.

Based on the results presented in tables 3, 4 and 5, one can conclude that:

1. The hot duplication of the one component, $\mathrm{m}=1$, increases $\mathrm{L}(0.1)$ from $0.1443 \mathrm{n}\left(\alpha_{1}+\alpha_{2}\right)$ to $0.1516 \mathrm{n}\left(\alpha_{1}+\alpha_{2}\right)$, see table 3 . The same effect on $\mathrm{L}(0.1)$ can occur by reducing the failure rates of:

(1.1) The second stage of: (i) one component, $r=1$, by the factor $\rho=0.3235$, (ii) two components, $r=2$, by the factor $\rho=0.6618$, (iii) three components, $r=3$, by the factor $\rho=$ 0.7745 , see table 4 .

(1.2) The first and second stages of: (i) one component, $r=1$, by the same factor $\rho=0.4895$, (ii) two components, $r=2$, by the same factor $\rho=0.7448$, (iii) three components, $r=2$, by the same factor $\rho=0.8298$, see table 5 .

Table 4. The SREF, $\rho_{2}^{H}(\gamma)$

\begin{tabular}{c|ccc|ccc|ccc}
\hline \hline \multirow{2}{*}{$\gamma$} & \multicolumn{3}{|c|}{$\mathrm{m}=1$} & \multicolumn{3}{c|}{$\mathrm{m}=2$} & \multicolumn{3}{c}{$\mathrm{m}=3$} \\
\cline { 2 - 9 } & $\mathrm{R}=1$ & $\mathrm{R}=2$ & $\mathrm{R}=3$ & $\mathrm{R}=1$ & $\mathrm{R}=2$ & $\mathrm{R}=3$ & $\mathrm{R}=1$ & $\mathrm{R}=2$ & $\mathrm{R}=3$ \\
\hline \hline 0.1 & 0.3235 & 0.6618 & 0.7745 & NA & 0.3944 & 0.5963 & NA & 0.1891 & 0.4594 \\
0.2 & 0.1955 & 0.5978 & 0.7318 & NA & 0.2835 & 0.5223 & NA & 0.0506 & 0.3670 \\
0.3 & 0.0926 & 0.5463 & 0.6975 & NA & 0.1934 & 0.4623 & NA & NA & 0.2928 \\
0.4 & NA & 0.4991 & 0.6660 & NA & 0.1096 & 0.4064 & NA & NA & 0.2243 \\
0.5 & NA & 0.4525 & 0.6350 & NA & 0.0258 & 0.3505 & NA & NA & 0.1564 \\
0.6 & NA & 0.4040 & 0.6027 & NA & NA & 0.2912 & NA & NA & 0.0848 \\
0.7 & NA & 0.3502 & 0.5668 & NA & NA & 0.2240 & NA & NA & 0.0047 \\
0.8 & NA & 0.2843 & 0.5229 & NA & NA & 0.1407 & NA & NA & NA \\
0.9 & NA & 0.1848 & 0.4565 & NA & NA & 0.0152 & NA & NA & NA \\
\hline \hline
\end{tabular}

Table 5. The SREF, $\rho^{H}(\gamma)$

\begin{tabular}{c|ccc|ccc|ccc}
\hline \hline \multirow{2}{*}{$\gamma$} & \multicolumn{3}{|c|}{$\mathrm{m}=1$} & \multicolumn{3}{c|}{$\mathrm{m}=2$} & & $\mathrm{~m}=3$ \\
& $\mathrm{R}=1$ & $\mathrm{R}=2$ & $\mathrm{R}=3$ & $\mathrm{R}=1$ & $\mathrm{R}=2$ & $\mathrm{R}=3$ & $\mathrm{R}=1$ & $\mathrm{R}=2$ & $\mathrm{R}=3$ \\
\hline \hline 0.1 & 0.4895 & 0.7448 & 0.8298 & 0.0753 & 0.5377 & 0.6918 & $\mathrm{NA}$ & 0.3743 & 0.5829 \\
\hline 0.2 & 0.4061 & 0.7031 & 0.8020 & $\mathrm{NA}$ & 0.4629 & 0.6419 & $\mathrm{NA}$ & 0.2784 & 0.518925 \\
\hline 0.3 & 0.3427 & 0.6714 & 0.7809 & NA & 0.4048 & 0.6032 & NA & 0.2036 & 0.4691 \\
\hline 0.4 & 0.2879 & 0.6439 & 0.7626 & NA & 0.3529 & 0.5686 & NA & 0.1367 & 0.4245 \\
\hline 0.5 & 0.2376 & 0.6188 & 0.7459 & NA & 0.3036 & 0.5357 & NA & 0.0724 & 0.3816 \\
\hline 0.6 & 0.1897 & 0.5949 & 0.7299 & NA & 0.2543 & 0.5028 & NA & 0.007 & 0.3381 \\
\hline 0.7 & 0.1429 & 0.5715 & 0.7143 & NA & 0.2030 & 0.4687 & NA & NA & 0.2916 \\
\hline 0.8 & 0.0963 & 0.5481 & 0.6988 & NA & 0.1473 & 0.4315 & NA & NA & 0.2387 \\
\hline 0.9 & 0.0489 & 0.5245 & 0.6829 & NA & 0.0829 & 0.3886 & NA & NA & 0.1709 \\
\hline \hline
\end{tabular}


2. In the same manner one can read the rest results.

3. The notation NA in tables 4 and 5 means that the value of SREF is not available and therefore there is possible equivalence between the system improved by HDM and that system improved by using the redundancy method at this level.

Table 6 introduces the values of the mean reliability equivalence factor, when we reduce the second stage by $\xi_{2}^{H}$ and the two stages by the same factor $\xi^{H}$.

Table 6. The MREF

\begin{tabular}{c|ccc|ccc}
\hline \hline \multirow{2}{*}{$\mathrm{r}$} & \multicolumn{3}{|c|}{$\xi_{2}^{H}$} & \multicolumn{3}{|c}{$\xi^{H}$} \\
\cline { 2 - 7 } & $\mathrm{m}=1$ & $\mathrm{~m}=2$ & $\mathrm{~m}=3$ & $\mathrm{~m}=1$ & $\mathrm{~m}=2$ & $\mathrm{~m}=3$ \\
\hline \hline & & & & & & \\
1 & $\mathrm{NA}$ & $\mathrm{NA}$ & $\mathrm{NA}$ & 0.2948 & $\mathrm{NA}$ & $\mathrm{NA}$ \\
2 & 0.4966 & 0.0765 & $\mathrm{NA}$ & 0.6474 & 0.3359 & 0.0732 \\
3 & 0.6644 & 0.3843 & 0.1659 & 0.7649 & 0.5573 & 0.3821 \\
\hline \hline
\end{tabular}

One can conclude that,

1. the improved system that can be obtained by improving the one component of the system components, $m=1$, according to HDM, has the same mean time to failure of that system which can be obtained by reducing:

(1.1) the second stage of (i) two components, $r=2$ by the factor, $\xi=0.4966$, (ii) three components, $r=3$ by the factor, $\xi=0.6644$,

(1.2) the two stages of (i) one component, $r=1$ by the same factor, $\xi=0.2948$, (ii) two components, $\mathrm{r}=2$ by the same factor, $\xi=0.6474$, (iii) three components, $r=3$ by the same factor, $\xi=0.7649$.

2. In the same manner one can read the rest results.

3. The notation NA in table 6 means that the value of MREF is not available and therefore there is possible equivalence between the system improved by HDM and that system improved by using the redundancy method at this level.

\subsection{Special Cases}

We can calculate the reliability equivalence factors for the special cases from the present system as follows:

1. if $\beta_{\mathrm{i}}=1$, the failure rates of the stages are constant. We have the mixture of the constant failure rates, in this case, the component with mixture failure rate has the Exponential distribution with parameter $\left(\frac{\theta_{1}}{\alpha_{1}}+\frac{\theta_{2}}{\alpha_{2}}\right)$.

2. if $\beta_{\mathrm{i}}=2$, the failure rates of the stages are increasing failure rates. we have the mixture of the increasing failure rates, equation $(20,21)$, can be reduced to

$$
h_{i}(t)=\frac{2}{\alpha_{i}^{2}} t, \quad R_{i}(t)=\exp \left\{-\left[\theta_{1}\left(\frac{t}{\alpha_{1}}\right)^{2}+\theta_{2}\left(\frac{t}{\alpha_{2}}\right)^{2}\right]\right\}
$$

In this case, the component with mixture failure rate has the Rayleigh distribution with parameter $\left(\frac{\theta_{1}}{\alpha_{1}^{2}}+\frac{\theta_{2}}{\alpha_{2}^{2}}\right)$.

Therefore, the reliability equivalence factor for the system with two stages of the failure rates, when the mixture failure rate of the components are exponential or Rayleigh distribution can be obtained as a special cases from the studied system in this section.

\section{Conclusions}

The quality of the system reliability can be improved using different methods of the system improvements. The results can be used to distinguish between the original and improved systems performances and calculate the equivalent between different cases of improving methods.

The reliability equivalence factor for a simple system consists of one component has two stages of lifetimes, with Weibull failure rates calculated and also for a system with n-identical components in series system, each component has two stages of the Weibull failure rate functions. Some special cases are derived.

\section{REFERENCES}

[1] Billinton, R and Allan, R., R (1983), Reliability Evaluation of Engineering Systems: Concepts and Techniques, Plenum Press, New York and London

[2] Elsayed, A.E. (1996), Reliability Engineering, Piscataway, New Jersey

[3] Kapur, K.C. and Lamberson, L.R. (1977), Reliability in Engineering Design, John Wiley

[4] Lewis, E.E. (1996), Introduction to Reliability Engineering, John Wiley \& Sons, Inc., New York, 2nd ed

[5] Mustafa, A. (2002), Reliability Equivalence of Some Systems, Master Thesis, Department of Mathematics, Faculty of Science, Mansoura University, Egypt.

http://www.mans.edu.eg/pcvs/3118/

[6] Mustafa, A. (2008). Reliability equivalence of Two Nonidentical Components. International Journal of Reliability and Applications. Vol. 9, No. 1, pp.79-93

[7] Mustafa A. and El-Bassoiuny A.H. (2008). Reliability equivalence of some systems with mixture linear increasing failure rates. Pakistan Journal of Statistics, Accepted

[8] Mustafa, A., El-Desouky, B. S. and El-Dawoody, M. (2007). Reliability Equivalence factor of Series System with Mixture Failure Rates. The 42th Annual Conference ISSR Cairo University 2-5 Dec., Egypt.Pp 190-210

[9] Mustafa, A., Sarhan, A. M. and Al-Ruzaiza, A. (2007), Reliability equivalence of a parallel-series system, Pakistan Journal of Statistics, Vol. 23(3), 241-254

[10] Råde, L. (1993a), Reliability equivalence, Microelectrons Reliability, 33, 323-325

[11] Råde, L. (1993b), Reliability survival equivalence, Microelectrons Reliability, 33, 881-894

[12] Sarhan, A.M. (2000), Reliability Equivalence of independent 
and non-identical components series systems, Reliability Engineering \& System Safety, 67, 293-300

[13] Sarhan, A.M. (2002), Reliability Equivalence with a basic series/parallel system, Applied Mathematics and Computation, 132, 115-133

[14] Sarhan A.M. (2004), Reliability equivalence factors of a bridge network system, International Journal of reliability and Applications, Vol. 2, 81-103

[15] Sarhan A.M. (2005), Reliability equivalence factors of a parallel system, Reliability Engineering \& System Safety, Vol. $87,405-411$

[16] Sarhan A.M. and Mustafa, A. (2006), Reliability equivalence of a series system consists of $\mathrm{n}$ independent and non-identical components, International Journal of Reliability and Applications, Vol. 7, No. 2, pp 111-125

[17] Sarhan, A. M., Tadj, L., Al-Khodari, A. and Mustafa, A. (2008). Equivalence factors of a parallel- series system, $A p$ plied Sciences, Vol.10, pp. 219-230

[18] Sarhan, A.M., Al-Ruzaiza, A.S., Alwasel, I.A. and El-Gohary, A. (2004), Reliability equivalence of a series-parallel system, Applied Mathematics and Computation, Vol. 154,257-277

[19] Xia, Y. and Zhang, G. (2007), Reliability equivalence factors in Gamma distribution, Applied Mathematics and Computation, Vol. 187, 567-573 